\title{
What is targeted proteomics? A concise revision of targeted acquisition and targeted data analysis in mass spectrometry
}

\author{
Eva Borràs ${ }^{1,2}$, Eduard Sabidó ${ }^{1,2, *}$
}

${ }^{1}$ Proteomics Unit, Centre de Regulació Genòmica (CRG), Barcelona Institute of Science and Technology (BIST), Dr. Aiguader 88, Barcelona, Barcelona

${ }^{2}$ Universitat Pompeu Fabra (UPF), Dr. Aiguader 88, Barcelona, Barcelona

Targeted proteomics has gained significant popularity in mass spectrometry-based protein quantification as a method to detect proteins of interest with high sensitivity, quantitative accuracy and reproducibility. ${ }^{1,2}$ Beyond its analytical capabilities, targeted proteomics has been described as a method to test specific hypotheses for a subset of proteins, in contrast with other mass spectrometry approaches, i. e. screening proteomics, in which thousands of proteins can be quantified without any prior knowledge. ${ }^{3}$

However, the emergence of a wide variety of so-called targeted proteomics methodssome of them with high-throughput capabilities-has introduced the use of different types of mass analyzers, acquisition modes, and data analysis strategies, which in several cases are common to well-established screening proteomics methods. It is for these reasons that some of the concepts associated to targeted proteomics need to be revisited, as targeted proteomics can no longer be linked to a particular type of instrument, nor to a specific acquisition method, nor to certain analytical capabilities. For instance, targeted proteomics methods are currently implemented in a variety of mass analyzers, including combinations of quadrupoles, orbital ion traps (orbitrap), and time-of-flight mass analyzers. Moreover, the emergence of methods that combine dataindependent acquisition with targeted data analysis has evidenced the capability of targeted proteomics to go far beyond the quantification of a few dozens of selected proteins, and several methods have reported thousands of peptides and proteins being quantified. ${ }^{4-6}$ In addition, with the flourishing of different targeted methods, a myriad of acronyms and commercial names have appeared to refer to them, which do not necessarily reflect the structure of the acquired data nor the strategies followed for data analysis.

With such diversity of names, data acquisition strategies and data analysis workflows it is easy to overlook the essence of each method and to determine what makes each of them a targeted proteomics method. Moreover, the increasing similarities in data structure and data analysis strategies that some recent targeted methods share with screening proteomics have introduced some additional confusion.

In this viewpoint, we revisit the main targeted proteomics methods and classify them into those that perform targeted data acquisition and those that are based on targeted

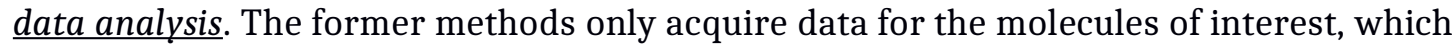
need to be defined prior to acquisition, whereas the latter acquire data for all molecular species and specific information is later extracted post-acquisition. In parallel, we establish a second orthogonal division to differentiate those targeted methods that are based on peptide ion data (i. e. methods that rely on the mass of an entire molecule or

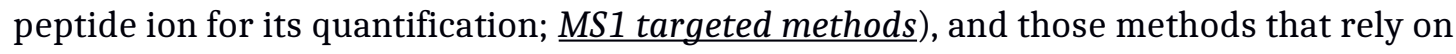

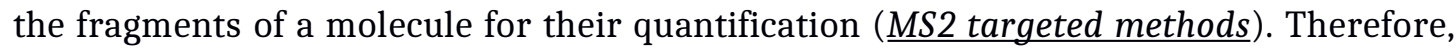


here we define four different categories of targeted approaches to classify the different targeted proteomics methods that are currently available in the field.

\section{Targeted acquisition at peptide ion level (MS1)}

\section{Selected ion monitoring}

Selected ion monitoring (SIM) is a mass spectrometry acquisition method in which only a small mass-to-charge range is transmitted to the detector. In this acquisition mode the mass analyzer is set to filter for one particular peptide ion, which is then constantly monitored over the time and its signal is used for quantification (Figure 1A). ${ }^{7-9}$ No peptide fragmentation is done in the mass spectrometer, though some methods can trigger a fragmentation event when the SIM signal exceeds a certain threshold and thus generate a MS2 spectrum for identification purposes. These methods are called SIMtriggered MS2 methods, in which the SIM signal is used for quantification purposes whereas the MS2 spectra is used for confirming the molecule identity (Figure 1B). ${ }^{10}$

However, peptide ions do not need to be monitored in a continuous form and operating the mass spectrometer with a sampling rate of a few data points per second is normally sufficient to reconstruct a peptide chromatographic peak for quantification purposes. The fact that chemical species do not need to be monitored constantly introduces a time gap between two consecutive sampling events during which the mass spectrometer can be used to sample another peptide of interest. This strategy enables monitoring several peptides within the same method and, therefore, it introduces peptide multiplexing capabilities to the SIM methods.

Selected ion monitoring (SIM) exhibits an improved dynamic range over methods that acquire the full mass range as the filtering step-usually performed with a quadrupole mass analyzer-isolates the peptide ions of interest, and transmits only a small mass-tocharge range to the detector. ${ }^{11}$

\section{Targeted data analysis at peptide ion level (MS1)}

\section{MS1 Extracted Ion Chromatograms}

Data-independent acquisition at the peptide level makes it possible to acquire peptide data for virtually all peptide ions present in a sample. In this strategy, a high-resolution mass analyzer-such as an orbitrap or a time-of-flight-is used to constantly sample the full mass range at the peptide level during the entire chromatographic gradient (Figure 2A). Full-range MS1 spectra sampled in regular intervals can then be aligned in the time axis to obtain the precursor ion chromatograms by targeted data analysis. Area under the curve is eventually calculated and used for peptide quantification (Figure 2B). Data generated by MS1 data-independent acquisition can also be represented as MS1 maps, which contain mass-to-charge, retention time, and intensity information for each peptide ion. ${ }^{12-14}$ However, these MS1 data lack peptide sequence information. Therefore, to perform peptide quantification, the retention time and mass-to-charge coordinates need to be extracted using reference isotopically-labeled internal standards, previous knowledge, or MS2 spectra acquired ad hoc for the most intense peptide ions or for a set of mass-to-charge ratios specified in an inclusion list.

It is worth mentioning that in this case the structure of the acquired data is similar - or in many cases even the same-as in screening proteomics. Quantitative data could thus 
be extracted for every single peptide in the sample without any prior peptide selection and, in fact, these MS1-maps have often been used for label-free quantification in screening proteomics methods. Therefore, what makes this method a targeted proteomics method is subtle but profound, and it is based on the concept of consistently extracting quantitative information only for the peptides of interest related to a given hypothesis. The same acquired data analyzed without any hypothesis, and with quantitative information for all available peptides would classify as a screening (nontargeted) proteomics experiment.

Methods that rely on targeted data analysis at the peptide level can provide quantitative information of virtually any peptide precursor ion. However, they exhibit high chemical noise due to the co-elution of high and low-abundant peptides when screening the entire mass range in complex samples. This phenomenon limits the dynamic range of these methods, and results in signals that often contain interferences from other peptide ions.

\section{Targeted data acquisition at fragment ion level (MS2)}

\section{Selected reaction monitoring}

Selected reaction monitoring (SRM) is one of the main strategies used in targeted proteomics experiments in which a set of peptides is selected to quantify a protein of interest. ${ }^{15-20}$ SRM is performed on triple quadrupole mass spectrometers in which the first mass analyzer is set to filtering mode with a narrow isolation window (e.g. $\pm 1 \mathrm{~m} / \mathrm{z}$ ) to isolate a specific peptide precursor ion of interest. Then, the isolated peptide is fragmented, and one of the resulting fragment ions is monitored with another mass analyzer set to filter a concrete mass-to-charge ratio. The double selection of a peptide precursor ion and peptide fragment ion is called a transition (Figure 3A). The probability of two peptide fragments having i) the same mass, ii) deriving from two different isobaric peptides, iii) and exhibiting the exact same retention time, is low. Therefore, the strategy of targeted acquisition using transitions is highly specific, it benefits from low chemical noise resulting in high sensitivity measurements, and it can detect proteins in complex samples as low as in a few copies per cell for selected molecules. ${ }^{21}$

Similarly to selected ion monitoring, SRM transitions can be used to trigger MS2 spectra for identification purposes given a certain intensity threshold. ${ }^{22,23}$ However, Selected reaction monitoring can sequentially monitor several fragment ions from the same peptide between two sampling events of the same transition. By monitoring several fragments of the same peptide precursor ion not only quantitative information is gathered, but also peptide identification can be inferred from the data. Typically, three to five fragment ions per peptide are measured, and one to three unique peptides with good quantitative properties are used for protein quantification (Figure $3 \mathrm{~B}$ ). ${ }^{24-26}$ The time between two sampling events of the same transition is the cycle time, and the time that the mass spectrometer dedicates to each transition is the dwell time. Therefore, the smaller the dwell time, the more independent sampling events will fit in a cycle.

The number of different transitions that can be monitored within a method depends on the chromatographic peak width and the sampling rate, which should provide a minimum of 8-10 fragment data points per chromatographic peak for accurate and precise quantification. The number of monitored transitions can be increased by limiting the measurement of each transition only around the peptide retention time, so that not all transitions within a method are recorded all the time. Since the method 
measures sequentially several fragment ions from different peptides, it is sometimes referred to as Multiple selection monitoring or MRM.

Compared to MS1 targeted acquisition methods (SIM), SRM enables both peptide identification and quantification simultaneously from the acquired data when several transitions are monitored for the same precursor. Moreover, it exhibits an improved dynamic range and sensitivity, as the double filtering step-at the precursor and the fragment level-results in an improved signal-to-noise ratio. However, as transitions need to be defined prior to acquisition, SRM only renders quantitative information of the selected peptides and fragments, and in case other peptides or fragment ions need to be explored, then, data need to be re-acquired.

\section{Parallel reaction monitoring}

Parallel reaction monitoring (PRM) is a targeted acquisition method that simultaneously analyzes all fragment ions of a pre-selected peptide of interest. ${ }^{27,28}$ In this method the first mass analyzer (a quadrupole) is set to filtering mode with a narrow isolation window (e.g. $1 \mathrm{~m} / \mathrm{z}$ ) to isolate the peptide ion of interest. Then the selected peptide is fragmented and the resulting fragment ions are simultaneously analyzed using a mass analyzer set to screening mode (Figure 3C). This strategy contrasts with Selected reaction monitoring, which performs sequential acquisition of each fragment from a given peptide as it needs to finish the sampling of one fragment before moving to the next one. Therefore, although both methods require a peptide selection prior mass spectrometric acquisition (targeted acquisition), PRM does not need fragment ions to be selected in advance. In PRM, targeted data analysis is used to retrieve the chromatographic peaks of those fragment ions that are most suitable for peptide identification and quantification i. $e$. those that have better signal-to-noise, those with no interferences, and/or those that provide more sequence information. Therefore, PRM has flexibility in selecting the fragment ions a posteriori, and in case some fragment ions show interferences, others can be re-extracted from the data without the need to reacquire the sample (Figure 3D). ${ }^{29-33}$

Parallel reaction monitoring only acquires information for the pre-selected peptides of interest (i. e. targeted acquisition), and similarly to SRM, it provides both identification and quantification information. PRM is normally done in quadrupole-orbitrap or quadrupole-time-of-flight hybrid instruments, and fragment ions are therefore acquired with high resolution and high mass accuracy, which results in an increased selectivity as compared to triple quadrupole instruments. ${ }^{34}$ Parallel reaction monitoring exhibits a high dynamic range, high signal-to-noise, and excellent sensitivity and specificity for peptide and protein quantification.

\section{Targeted data analysis at fragment ion level (MS2)}

Since several years, there exist different data-independent acquisition methods at the fragment ion level that acquire all fragment ions from virtually any peptide in a sample. ${ }^{35-41}$ Compared to targeted acquisition methods such as SIM, PRM and SRM, these methods acquire data for all peptides and therefore, the targets of interest can be selected post-acquisition.

These methods rely on the use of one or several broadband isolation windows that isolate all peptide ions in a sample within each cycle. 
All peptide ions co-isolated within a window are simultaneously fragmented, and the resulting fragment ion maps can be subjected to targeted data analysis to identify the co-eluting fragment ion groups from the peptides of interest. Identification of fragment ions group and peptide precursors rely on parameters such as retention time, fragment ion relative intensity, correlation with reference spectral libraries, and co-elution with internal reference peptides, among others (Figure 4) ${ }^{42-45}$ Isolation windows differ in multiple parameters depending on the multiple variants that currently exist for MS2 data-independent acquisition..$^{41,46,47}$ In this regard, isolation windows i) exhibit different widths-ranging from $2-3 \mathrm{~m} / \mathrm{z}$ to the full mass range-, ii) are acquired either sequentially or in a non-consecutive order, and iii) are either juxtaposed or overlapped.

MS2 data-independent acquisition methods are usually implemented in quadrupoleorbital ion traps, and quadrupole-time-of-flight hybrid mass analyzers. These methods provide both identification and quantification information of fragment and peptide ions with an improved dynamic range compared with their MS1 counterparts due to a better signal-to-noise ratio.

Data-independent acquisition methods at MS2 level generate datasets that contain fragment ions from all peptide precursors in the sample, and therefore can be queried for the peptides and fragments of interest after acquisition. As there is no limitation in which peptides to extract, data-independent acquisition methods at the fragment level can only be considered targeted as long as there is a hypothesis to be tested i. e. a set of proteins (can be many) are queried to extract their specific quantification information. The selected proteins/peptides can correspond to different protein isoforms, splicing variants, and specific modified peptides, ${ }^{48,49}$ but there needs to be a specific question to be answered. Without this premise, MS2 data-independent acquisition methods are not targeted per se, because the same acquired data can be analyzed with non-targeted data analysis strategies to extract the information of all proteins present in the sample, which would then classify as screening proteomics. ${ }^{50,51}$

\section{Wrap-up}

In the last years, targeted strategies on data acquisition and analysis have experienced an important expansion, and new methods are constantly being proposed sustained by a parallel improvement of instrument capabilities, computational workflows and new brilliant ideas. Here, we have classified the current existing methods into those that perform targeted data acquisition and those that are based on targeted data analysis, and we have differentiated them according to whether they rely on the mass of peptide ions (MS1 targeted methods), or on the fragments of the studied molecules (MS2 targeted methods).

Given the rapid development in the field, it is expected that differences between targeted and screening approaches will be progressively reduced, as soon it will be possible to target every peptide within a single acquisition method. ${ }^{52}$ Therefore, the analytical benefits of the current targeted acquisition methods-such as SRM and PRM - will be exploited in data-independent methods, and the differences in the data structure between targeted and screening proteomics will blur. Convergence might also affect data analysis workflows, with approaches that systematically perform targeted data analysis of all existing peptides, independently of the research question. Therefore, the discussed dichotomy between targeted and screening proteomics will probably be transferred from data acquisition and analysis to how researchers retrieve and treat information from pre-extracted datasets acquired with a universal "target-all" method. 



\section{Acknowledgements}

The CRG/UPF Proteomics Unit is part of the "Plataforma de Recursos Biomoleculares y Bioinformáticos (ProteoRed)" supported by grant PT13/0001 of the Instituto de Salud Carlos III (ISCIII) and the Spanish Ministry of Economy and Competitiveness. We acknowledge support from the Spanish Ministry of Economy and Competitiveness, “Centro de Excelencia Severo Ochoa 2013-2017", SEV-2012-0208, and "Secretaria d'Universitats i Recerca del Departament d'Economia i Coneixement de la Generalitat de Catalunya" (2014SGR678). 


\section{Bibliography}

1. Aebersold, R., Bensimon, A., Collins, B. C., Ludwig, C. and Sabido, E. (2016). Applications and developments in targeted proteomics: from SRM to DIA/SWATH. Proteomics 16, 2065-2067.

2. Marx, V. (2013). Targeted proteomics. Nature Methods 10, 19.

3. Picotti, P., Bodenmiller, B. and Aebersold, R. (2013). Proteomics meets the scientific method. Nature Methods 10, 24-27.

4. Liu, Y., Huettenhain, R., Surinova, S., Gillet, L. C., Mouritsen, J., Brunner, R., Navarro, P., and Aebersold, R. (2013). Quantitative measurements of N-linked glycoproteins in human plasma by SWATH-MS. Proteomics, 13(8), 1247-1256.

5. Liu, Y., Chen, J., Sethi, A., Li, Q. K., Chen, L., Collins, B., Gillet, L., Wollscheid, B., Zhang, H., and Aebersold, R. (2014). Glycoproteomic analysis of prostate cancer tissues by SWATH mass spectrometry discovers $\mathrm{N}$-acylethanolamine acid amidase and protein tyrosine kinase 7 as signatures for tumor aggressiveness. Molecular \& Cellular Proteomics, 13(7), 1753-1768.

6. Selevsek, N., Chang, C. Y., Gillet, L. C., Navarro, P., Bernhardt, O. M., Reiter, L., Cheng, L-Y., Vitek, O., and Aebersold, R. (2015). Reproducible and consistent quantification of the Saccharomyces cerevisiae proteome by SWATH-mass spectrometry. Molecular \& Cellular Proteomics, 14(3), 739-749.

7. Gatlin, C. L., Kleemann, G. R., Hays, L. G., Link, A. J., and Yates, J. R. (1998). Protein identification at the low femtomole level from silver-stained gels using a new fritless electrospray interface for liquid chromatography-microspray and nanospray mass spectrometry. Analytical biochemistry, 263(1), 93-101.

8. Haas, W., Faherty, B. K., Gerber, S. A., Elias, J. E., Beausoleil, S. A., Bakalarski, C. E., Li, X., Villén J. and Gygi, S. P. (2006). Optimization and use of peptide mass measurement accuracy in shotgun proteomics. Molecular \& Cellular Proteomics, 5(7), 1326-1337.

9. Streng, A. S., de Boer, D., Bouwman, F. G., Mariman, E. C., Scholten, A., van Dieijen-Visser, M. P., and Wodzig, W. K. (2016). Development of a targeted selected ion monitoring assay for the elucidation of protease induced structural changes in cardiac troponin T. Journal of proteomics, 136, 123-132.

10. Thomas, A., Walpurgis, K., Tretzel, L., Brinkkötter, P., Fichant, E., Delahaut, P., Schanzer, W., and Thevis, M. (2015). Expanded test method for peptides $>2 \mathrm{kDa}$ employing immunoaffinity purification and LC-HRMS/MS. Drug testing and analysis, 7(11-12), 990-998.

11. Banks, J. F. (1996). High-sensitivity peptide mapping using packed-capillary liquid chromatography and electrospray ionization mass spectrometry. Journal of Chromatography A, 743(1), 99-104.

12. Silva, J. C., Denny, R., Dorschel, C. A., Gorenstein, M., Kass, I. J., Li, G. Z., McKenna, T., Nold, M. J., Richardson, K., Young, P., and Geromanos, S. (2005). Quantitative proteomic analysis by accurate mass retention time pairs. Analytical Chemistry, 77(7), 2187-2200.

13. Shen, X., Shen, S., Li, J., Hu, Q., Nie, L., Tu, C., Wang, X., Orsburn, B., Wang, J., and Qu, J. (2017). An IonStar Experimental Strategy for MS1 Ion Current-Based Quantification Using UltrahighField Orbitrap: Reproducible, In-Depth, and Accurate Protein Measurement in Large Cohorts. Journal of Proteome Research. Doi: 10.1021/acs.jproteome.7b00061

14. Schilling, B., Rardin, M. J., MacLean, B. X., Zawadzka, A. M., Frewen, B. E., Cusack, M. P., Sorensen, D. J., Bereman, M. S., Jing, E., Wu, C. C., Verdin, E., Kahn, C. R., MacCoss, M. J. and Verdin, E. (2012). Platform-independent and label-free quantitation of proteomic data using MS1 extracted ion chromatograms in Skyline application to protein acetylation and phosphorylation. Molecular \& Cellular Proteomics, 11(5), 202-214.

15. Drabovich, A. P., Jarvi, K., and Diamandis, E. P. (2011). Verification of male infertility biomarkers in seminal plasma by multiplex selected reaction monitoring assay. Molecular \& Cellular Proteomics, 10(12), M110-004127.

16. Drabovich, A. P., Pavlou, M. P., Dimitromanolakis, A., and Diamandis, E. P. (2012). Quantitative analysis of energy metabolic pathways in MCF-7 breast cancer cells by selected reaction monitoring assay. Molecular \& Cellular Proteomics, 11(8), 422-434.

17. Korbakis, D., Brinc, D., Schiza, C., Soosaipillai, A., Jarvi, K., Drabovich, A. P., and Diamandis, E. P. (2015). Immunocapture-selected reaction monitoring screening facilitates the development of ELISA for the measurement of native TEX101 in biological fluids. Molecular \& Cellular Proteomics, 14(6), 1517-1526.

18. Borràs, E., Cantó, E., Choi, M., Villar, L. M., Álvarez-Cermeño, J. C., Chiva, C., Montalban, X., Vitek, O., Comabella, M., and Sabidó, E. (2016). Protein-based classifier to predict conversion 
from clinically isolated syndrome to multiple sclerosis. Molecular \& Cellular Proteomics, 15(1), 318-328.

19. Duriez, E., Masselon, C. D., Mesmin, C., Court, M., Demeure, K., Allory, Y., Núria Malats, N., Matondo, M., Radvanyi, F., Garin, J., and Domon, B. (2017). Large-Scale SRM Screen of Urothelial Bladder Cancer Candidate Biomarkers in Urine. Journal of Proteome Research, 16(4), 1617-1631.

20. Lange, V., Picotti, P., Domon, B., and Aebersold, R. (2008). Selected reaction monitoring for quantitative proteomics: a tutorial. Molecular systems biology, 4(1), 222.

21. Picotti, P., Bodenmiller, B., Mueller, L. N., Domon, B. and Aebersold, R. (2009) Full dynamic range proteome analysis of $S$. cerevisiae by targeted proteomics. Cell 138, 795-806.

22. Unwin, R. D., Griffiths, J. R., Leverentz, M. K., Grallert, A., Hagan, I. M., \& Whetton, A. D. (2005). Multiple reaction monitoring to identify sites of protein phosphorylation with high sensitivity. Molecular \& Cellular Proteomics, 4(8), 1134-1144.

23. Picotti, P., Rinner, O., Stallmach, R., Dautel, F., Farrah, T., Domon, B., Wenschuh, H., and Aebersold, R. (2010). High-throughput generation of selected reaction-monitoring assays for proteins and proteomes. Nature Methods, 7(1), 43-46.

24. Worboys, J. D., Sinclair, J., Yuan, Y., and Jørgensen, C. (2014). Systematic evaluation of quantotypic peptides for targeted analysis of the human kinome. Nature Methods, 11(10), 1041-1044.

25. Kusebauch, U., Campbell, D. S., Deutsch, E. W., Chu, C. S., Spicer, D. A., Brusniak, M. Y., Slagel, J., Sun, Z., Stevens, J., Grimes, B., Shteynberg, D., Hoopmann, M. R., Blattmann, P., Ratushny, A. V., Rinner, O., Picotti, P., Carapito, C., Huang, C-Y., Kapousouz, M., Lam, H., Tran, T., Demir, E., Aitchison, J. D., Sander, C., Hood, L., Aebersold, R., and Moritz, R. L. (2016). Human SRMAtlas: A resource of targeted assays to quantify the complete human proteome. Cell, 166(3), 766.

26. Chiva, C., and Sabidó, E. (2017). Peptide selection for targeted protein quantitation. Journal of proteome research, 16(3), 1376-1380.

27. Peterson, A. C., Russell, J. D., Bailey, D. J., Westphall, M. S. and Coon, J. J. (2012). Parallel reaction monitoring for high resolution and high mass accuracy quantitative, targeted proteomics. Molecular \& Cellular Proteomics 11, 1475-1488.

28. Gallien, S., Duriez, E., Crone, C., Kellmann, M., Moehring, T., \& Domon, B. (2012). Targeted proteomic quantification on quadrupole-orbitrap mass spectrometer. Molecular \& Cellular Proteomics, 11(12), 1709-1723.

29. Martinez-Garcia, E., Lesur, A., Devis, L., Campos, A., Cabrera, S., van Oostrum, J., Matias-Guiu, X., Gil-Moreno, A., Reventos, J., and Domon, B. (2016). Development of a sequential workflow based on LC-PRM for the verification of endometrial cancer protein biomarkers in uterine aspirate samples. Oncotarget, 7(33), 53102.

30. Lawrence, R. T., Searle, B. C., Llovet, A., and Villén, J. (2016). Plug-and-play analysis of the human phosphoproteome by targeted high-resolution mass spectrometry. Nature Methods, 13(5), 431-434.

31. Henderson, C. M., Bollinger, J. G., Becker, J. O., Wallace, J. M., Laha, T. J., MacCoss, M. J., \& Hoofnagle, A. N. (2017). Quantification by nano liquid chromatography parallel reaction monitoring mass spectrometry of human apolipoprotein A-I, apolipoprotein B, and hemoglobin A1c in dried blood spots. Proteomics-Clinical Applications, doi: 10.1002/prca.201600103.

32. Gámez-Pozo, A., Trilla-Fuertes, L., Prado-Vázquez, G., Chiva, C., López-Vacas, R., Nanni, P., Berges-Soria, J., Grossmann, J., Díaz-Almirón, M., Ciruelos, E., Sabidó, E., Espinosa, E., and Fresno-Vara, J. A. (2017). Prediction of adjuvant chemotherapy response in triple negative breast cancer with discovery and targeted proteomics. PloS one, 12(6), e0178296.

33. Bilan, V., Selevsek, N., Kistemaker, H. A., Abplanalp, J., Feurer, R., Filippov, D. V., and Hottiger, M. O. (2017). New quantitative mass spectrometry approaches reveal different ADPribosylation phases dependent on the levels of oxidative stress. Molecular $\&$ Cellular Proteomics, 16(5), 949-958.

34. Gallien, S., Duriez, E., Demeure, K., and Domon, B. (2013). Selectivity of LC-MS/MS analysis: implication for proteomics experiments. Journal of proteomics, 81, 148-158.

35. Venable, J. D., Dong, M. Q., Wohlschlegel, J., Dillin, A., and Yates, J. R. (2004). Automated approach for quantitative analysis of complex peptide mixtures from tandem mass spectra. Nature Methods, 1(1), 39-45. 
36. Silva, J. C., Gorenstein, M. V., Li, G. Z., Vissers, J. P., and Geromanos, S. J. (2006). Absolute quantification of proteins by LCMSE a virtue of parallel MS acquisition. Molecular \& Cellular Proteomics, 5(1), 144-156.

37. Ramos, A. A., Yang, H., Rosen, L. E., and Yao, X. (2006). Tandem parallel fragmentation of peptides for mass spectrometry. Analytical Chemistry, 78(18), 6391-6397.

38. Panchaud, A., Scherl, A., Shaffer, S. A., Von Haller, P. D., Kulasekara, H. D., Miller, S. I., and Goodlett, D. R. (2009). Precursor acquisition independent from ion count: how to dive deeper into the proteomics ocean. Analytical Chemistry, 81(15), 6481-6488.

39. Geiger, T., Cox, J., and Mann, M. (2010). Proteomics on an Orbitrap benchtop mass spectrometer using all-ion fragmentation. Molecular \& Cellular Proteomics, 9(10), 22522261.

40. Gillet, L. C., Navarro, P., Tate, S., Röst, H., Selevsek, N., Reiter, L., Bonner, R., and Aebersold, R. (2012). Targeted data extraction of the MS/MS spectra generated by data-independent acquisition: a new concept for consistent and accurate proteome analysis. Molecular $\mathcal{E}$ Cellular Proteomics, 11(6), 0111-016717.

41. Egertson, J. D., Kuehn, A., Merrihew, G. E., Bateman, N. W., MacLean, B. X., Ting, Y. S., Canterbury, J. D., Marsh, D. M., Kellmann, M., Zabrouskov, V., Wu, C. C., and MacCoss, M. J. (2013). Multiplexed MS/MS for improved data-independent acquisition. Nature Methods, 10(8), 744-746.

42. Carvalho, P. C., Han, X., Xu, T., Cociorva, D., Carvalho, M. D. G., Barbosa, V. C., \& Yates III, J. R. (2010). XDIA: improving on the label-free data-independent analysis. Bioinformatics, 26(6), 847-848.

43. Bern, M., Finney, G., Hoopmann, M. R., Merrihew, G., Toth, M. J., \& MacCoss, M. J. (2009). Deconvolution of mixture spectra from ion-trap data-independent-acquisition tandem mass spectrometry. Analytical Chemistry, 82(3), 833-841.

44. Röst, H. L., Rosenberger, G., Navarro, P., Gillet, L., Miladinović, S. M., Schubert, O. T., Wolski, W., Collins, B. C., Malmström, J., Malmström L., and Aebersold, R. (2014). OpenSWATH enables automated, targeted analysis of data-independent acquisition MS data. Nature Biotechnology, 32(3), 219-223.

45. Röst, H. L., Liu, Y., D'Agostino, G., Zanella, M., Navarro, P., Rosenberger, G., Collins, B. C., Gillet, L., Testa, G., Malmström, L., Aebersold R. (2016). TRIC: an automated alignment strategy for reproducible protein quantification in targeted proteomics. Nature methods, 13(9), 777-783.

46. Anjo, S. I., Santa, C. and Manadas, B. (2017). SWATH-MS as a tool for biomarker discoveryfrom basic research to clinical applications. Proteomics. Doi: 10.1002/pmic.201600278

47. Chapman, J. D., Goodlett, D. R., and Masselon, C. D. (2014). Multiplexed and data-independent tandem mass spectrometry for global proteome profiling. Mass Spectrometry Reviews. 33, 452-470.

48. Sidoli, S., Fujiwara, R., and Garcia, B. A. Multiplexed data independent acquisition (MSX-DIA) applied by high resolution mass spectrometry improves quantification quality for the analysis of histone peptides. (2016). Proteomics 16, 2095-2105.

49. Sidoli, S., Lin, S., Xiong, L., Bhanu, N. V., Karch, K. R., Johansen, E., Hunter, C., Mollah, S., and Garcia, B. A. (2015). SWATH analysis for characterization and quantification of histone posttranslational modifications. Molecular \& Cellular Proteomics, mcp.0114.046102.

50. Tsou, C. C., Avtonomov, D., Larsen, B., Tucholska, M., Choi, H., Gingras, A. C., \& Nesvizhskii, A. I. (2015). DIA-Umpire: comprehensive computational framework for data-independent acquisition proteomics. Nature Methods, 12(3), 258-264.

51. Tsou, C.-C., Tsai, C.-F., Teo, G. C., Chen, Y.-J., and Nesvizhskii, A. I. (2016). Untargeted, spectral library-free analysis of data-independent acquisition proteomics data generated using Orbitrap mass spectrometers. Proteomics 16, 2257-2271.

52. Kang, Y., Burton, L., Lau, A., and Tate, S. (2017). SWATH-ID: An instrument method which combines identification and quantification in a single analysis. Proteomics, doi:10.1002/pmic.201500522 


\section{Figures}

Figure 1: Schematic representation of targeted acquisition methods at peptide ion level (MS1). A) Selected ion monitoring (SIM) uses a mass analyzer in filtering mode (e. g. 1-2 $\mathrm{m} / \mathrm{z}$ window) to isolate and monitor the signal of a selected peptide ion of interest (in red). B) The SIM signal can be further confirmed in hybrid mass spectrometers by SIMtriggered MS2 to obtain peptide sequence information.

Figure 2: A schematic representation of targeted data analysis methods at peptide ion level (MS1). A) Targeted data analysis methods at the peptide level rely on MS1 dataindependent acquisition often followed by a set of MS2 spectra triggered of the most intense peptide ions, which all together conform a cycle (black bar). B) MS1 spectra can be aligned along the time axis and peptide ion chromatograms are obtained by targeted data analysis to build the MS1 maps with retention time and $\mathrm{m} / \mathrm{z}$ coordinates.

Figure 3: Schematic representation of targeted data acquisition methods at fragment ion level (MS2). A) Selected reaction monitoring (SRM) uses a double filter to monitor a fragment ion (in purple) from a specific selected peptide (in red). B) Selected reaction monitoring sequentially monitors several fragment ions from the same peptide or from other precursors in each cycle. C) Parallel reaction monitoring combines peptide isolation (in red) using a filtering mass analyzer with the simultaneous analysis of all fragments with a second mass analyzer set to screening mode (represented in different tones of red). This method can sequentially monitor several peptides within each cycle represented in the figure as red and blue precursor ions. D) Targeted data analysis of the acquired MS2 spectra enables obtaining groups of co-eluting fragment ion chromatograms for each peptide precursor.

Figure 4: A schematic representation of targeted data analysis methods at fragment ion level (MS2). A) Targeted data analysis methods at the fragment level rely on MS2 dataindependent acquisition. Broad isolation windows at the precursor level result in complex full-range MS2 spectra sampled in regular time intervals. Here, an isolation scheme of 25-Da windows is illustrated, which cover the precursor mass range (MS1) from 400 to $1200 \mathrm{~m} / \mathrm{z}$ in 32 steps. The time required to cover the precursor full mass range-either in one large isolation window or as a result of merging multiple small or mid-size isolation windows-is the cycle time. B) Highly complex MS2 maps are aligned in the time axis and fragment ion chromatograms are obtained by targeted data analysis. 
Figure 1

A - peptide of interest
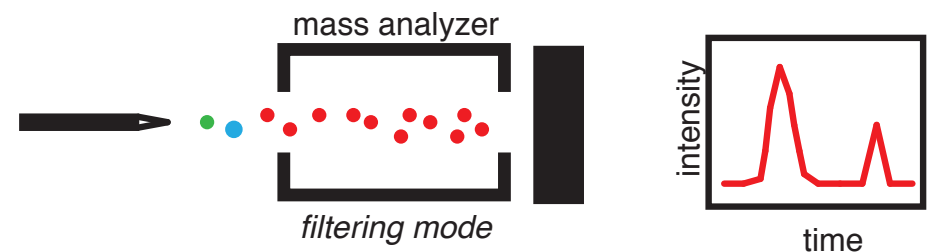

B

- peptide of interest
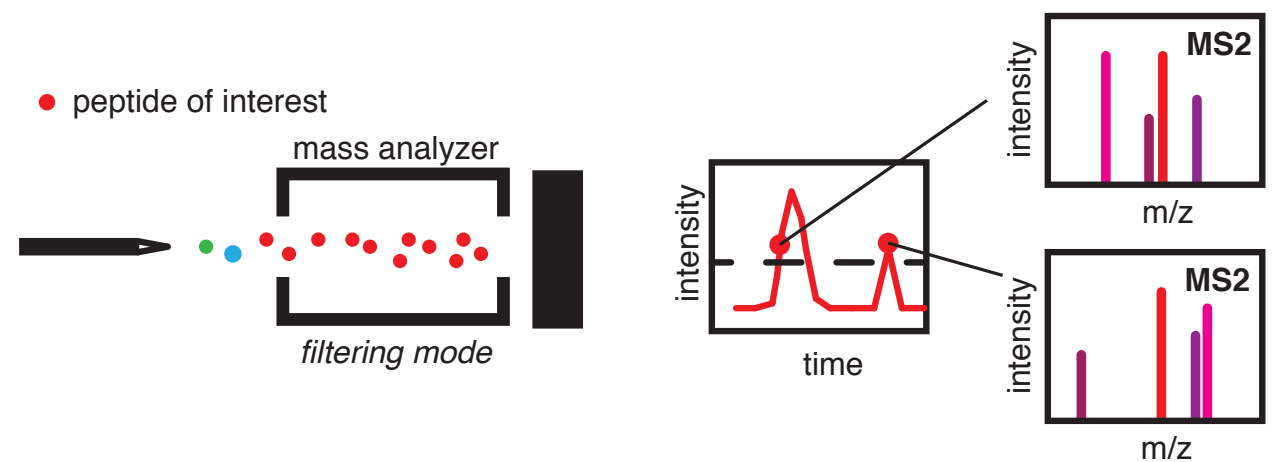
Figure 2

A

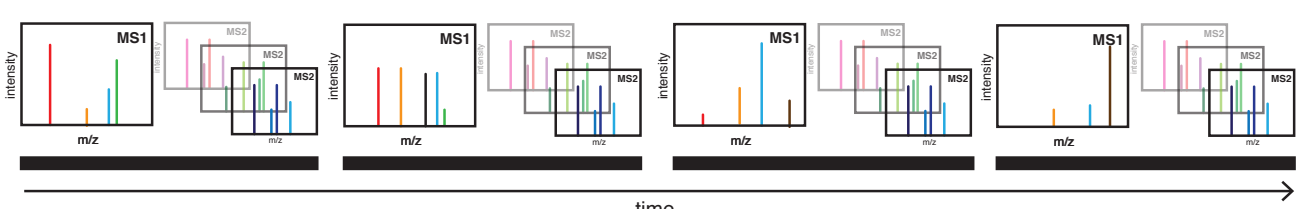

B

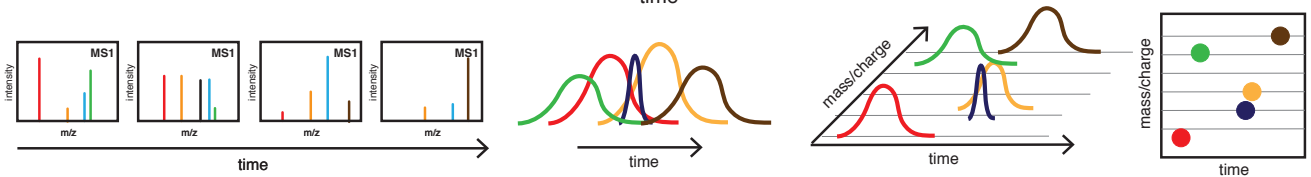


Figure 3

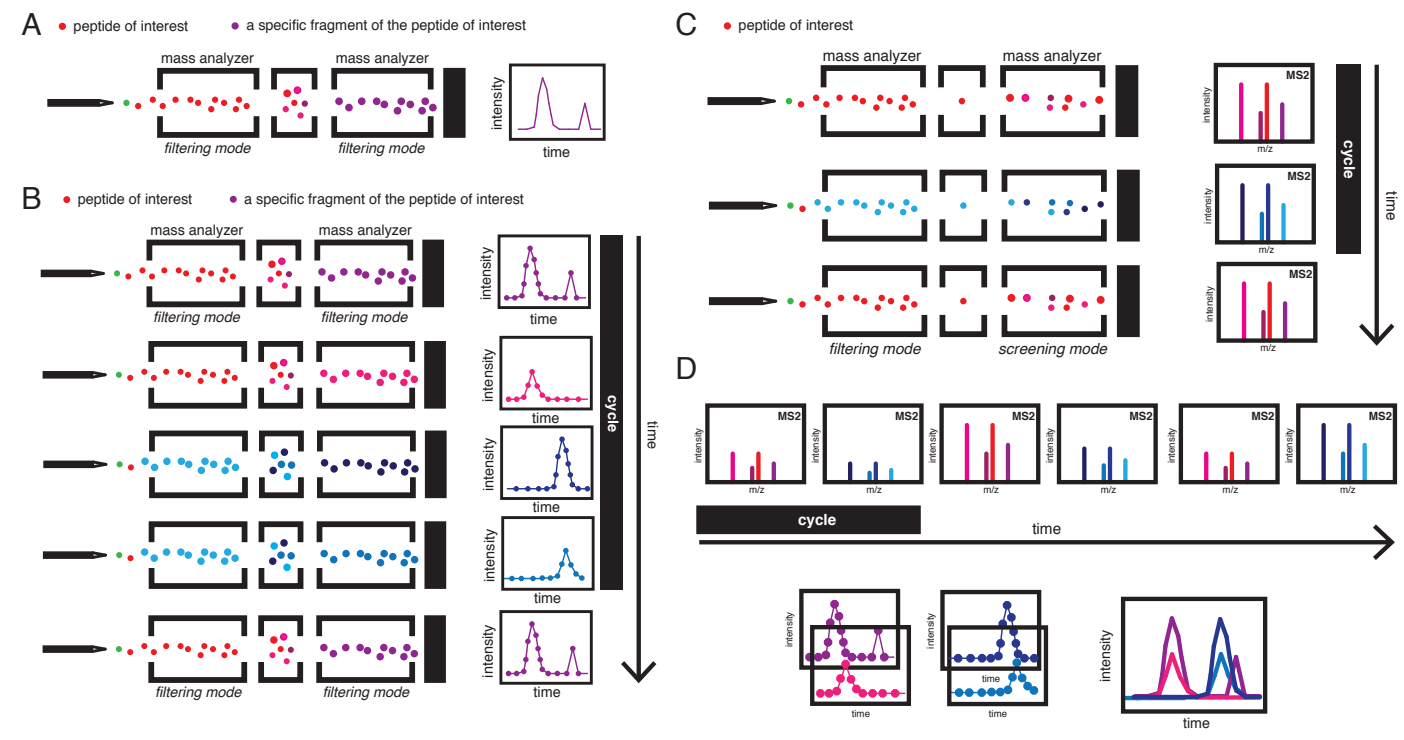


Figure 4

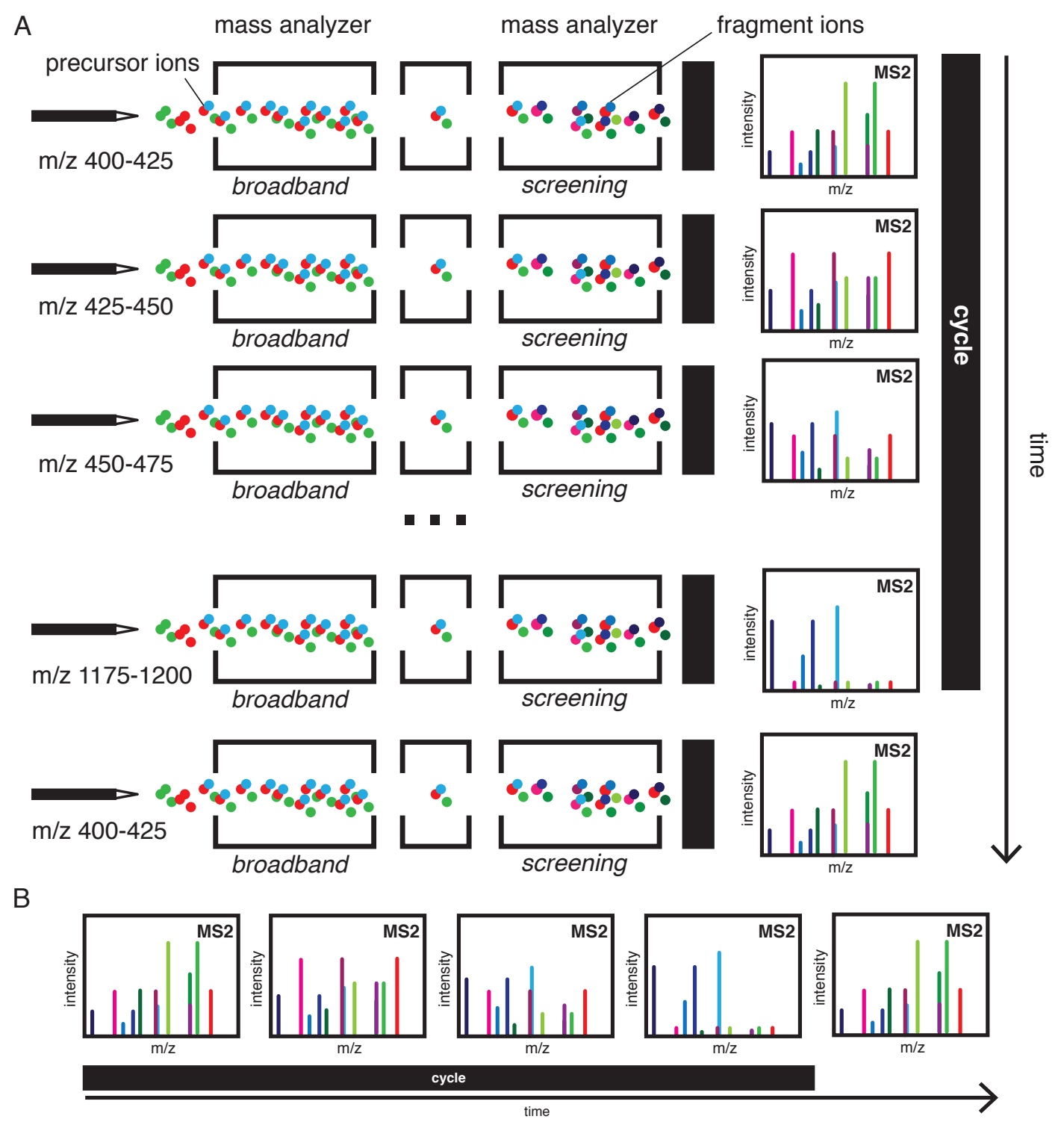

